\title{
Moving Medicine Out of the Clinic
}

\author{
Engineer John Ho develops wireless technologies for monitoring signals \\ produced by the body, hoping that the devices he creates will enable \\ clinicians to diagnose and treat diseases remotely.
}

\author{
By Katherine Wright
}

$\int$ ohn Ho likes making things. An engineer by training, the National University of Singapore researcher applies physics to the design of medical devices with new or improved capabilities. By leveraging previously unexploited behaviors of materials, he creates devices that push the envelope on how medical professionals can monitor patients' bodies while also being relatively simple to operate, he says.

Ho's inventions include clothing that can track physiological information, such as a person's posture or their temperature, tiny wireless implants that can stimulate nerves to treat disorders, and a network of devices that can wirelessly monitor the blood oxygen levels in thousands of patients. He hopes that these devices will help treat and diagnose diseases more quickly and effectively. Physics spoke to Ho to find out more

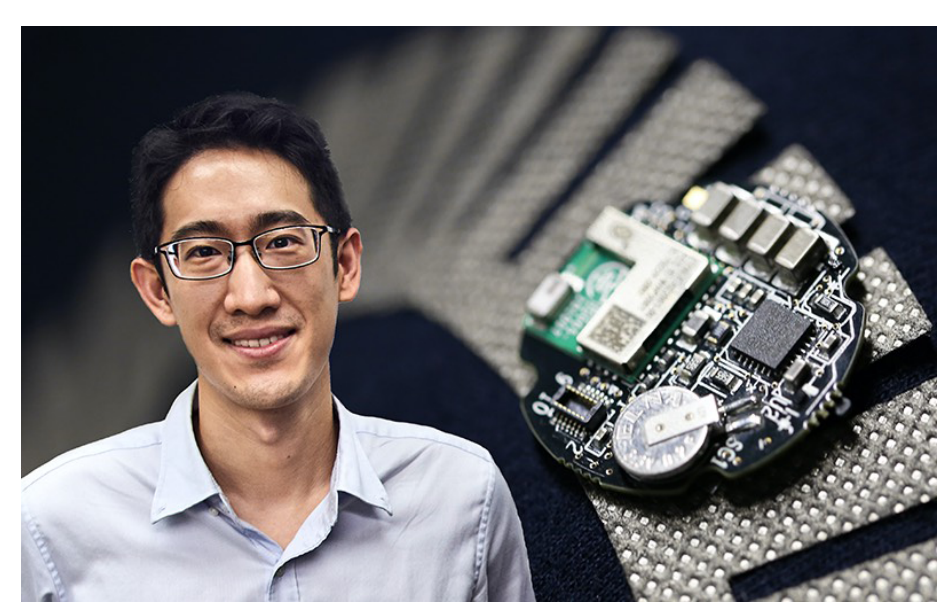

Credit: John Ho; APS/Carin Cain about how his devices work, how they might assist medical providers, and why we all might soon be wearing Wi-Fi enabled T-shirts.

All interviews are edited for brevity and clarity.

\section{What attracted you to engineering medical devices?}

We traditionally think of medicine as a field where clinicians fix ailments with pharmaceuticals. But what if we could instead build devices to treat and diagnose diseases? If we can make those devices and then operate them wirelessly, clinicians could remotely spot a problem and intervene. Medicine would no longer be confined to clinics, opening up treatments to more people, and clinicians could be much more proactive, potentially spotting future problems-like heart attacks-before they even happen.

\section{What are the biggest challenges in creating medical devices?}

One problem is power efficiency: the amount of energy available to a device ultimately determines what it can and can't do. With more energy, a device could do more "things"-make more calculations, monitor more variables, and maybe even perform small surgeries directly inside the body. Building devices that are power efficient and that stay on for a long time is a critical challenge.

Size is another big problem. Ideally, these devices should be small enough that clinicians could just inject them into the body. Today's devices are much bigger than that-the most commonly used pacemaker is about the size of the palm of my 


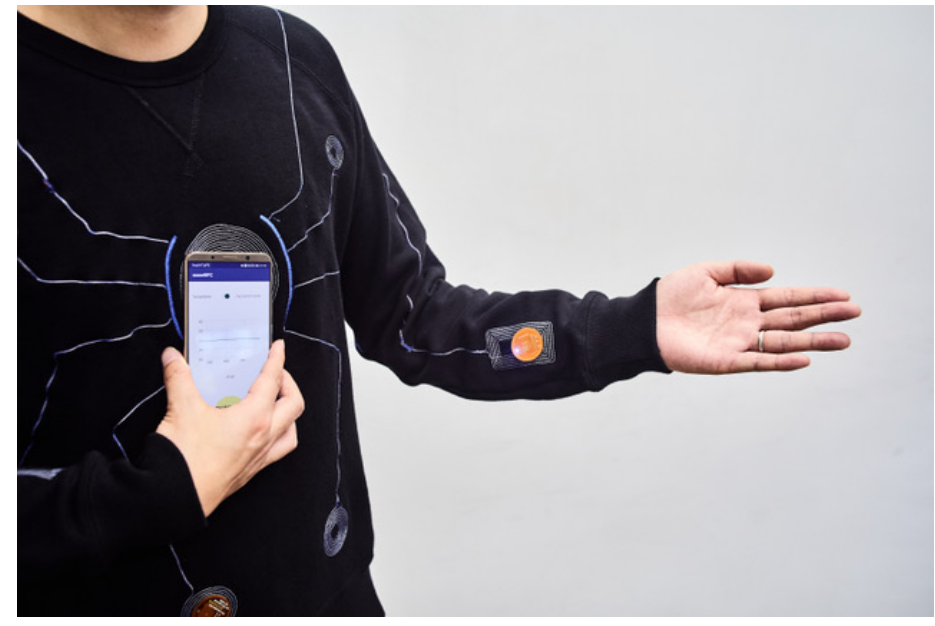

John Ho and his colleagues have developed a shirt that allows multiple sensors on the body to communicate with each other and with a smart phone.

Credit: J. Ho/National University of Singapore

hand-and they typically require invasive surgery to put them in place. That large size limits where these devices can be used and what they can be used for-you can't place a palm-sized device in the spinal cord, for example, where you might want to monitor electrical signals.

\section{What limits the size of a medical device?}

Today the bottleneck to shrinking a medical device is either its antenna or its battery, both of which are typically large. One way to get around the battery problem is to use wireless power transfer, which is the approach that I use. The devices I develop use both wireless power transfer and wireless communication.

\section{How does wireless power transfer work?}

For medical implants, wireless power transfer works by sending energy from the outside of the body to the device inside of the body. A transmitter on the skin above the implant emits energy in the form of radio-frequency fields, sound, or light to a receiver on the implanted device. The receiver captures the energy and converts it into electrical power.

\section{Tell me about your wireless shirt.}

Single devices, such as a watch, can capture what is happening at one part of the body. We wanted to find a way to use multiple devices to gain a picture of what the whole body is doing. A central challenge in achieving that goal is getting the devices to talk to each other, a problem we solve with wireless communication.

Using wireless signals, a device on the back of a body can talk to a device on the front of the body if the devices are correctly connected. Wireless signals tend to just spread out in space from their point of origin. So we had to find a way to confine the signals and then direct them over the surface of a body to a specific point. We do that with conductive fabric patterns stuck onto a regular T-shirt.

The tracks of the pattern "trap" the wireless signals from each device and then move them to where we want them to go-you can think of the patterns as a waveguide for the signals.

\section{What kind of sensors might you connect to each other with such a shirt?}

An obvious one would be an electrocardiogram (ECG) sensor that measures the electrical activity of the heart. Another might be a pulse oximeter, which uses light to measure the amount of oxygen in the blood. Those things can all be measured in a clinic using devices connected by wires to batteries and monitors. We imagine instead having the sensors stuck to the body with stickers. The clothing would offer a way to power devices, collect their data, and send that data to a clinician, say via a phone. The wearer would be able to move around completely unencumbered of wires and could be monitored anytime, anywhere.

Katherine Wright is the Deputy Editor of Physics. 